\title{
Modelo do Fluxo Biológico do Fósforo de Fontes de Fosfato em Suínos, Usando o ${ }^{32} \mathbf{P}$ como Marcador ${ }^{1}$
}

\author{
João Batista Lopes ${ }^{2}$, Dorinha Miriam Silber Schmidt Vitti ${ }^{3}$, Adibe Luiz Abdalla ${ }^{3}$, \\ Marinéia de Lara Haddad ${ }^{4}$, Agustinho Valente de Figueirêdo ${ }^{2}$, Regina Célia Botéquio de Moraes ${ }^{5}$
}

RESUMO - A pesquisa destinou-se a simular modelos matemáticos relacionados ao fluxo de fósforo (P), partindo-se das hipóteses de que o P endógeno do trato digestivo foi absorvido na mesma proporção do $\mathrm{P}$ alimentar e o fluxo de entrada e de saída desse mineral nos compartimentos foi constante. Foram avaliadas as variáveis absorção, retenção, $\mathrm{P}$ endógeno que retorna ao trato gastrintestinal, $\mathrm{P}$ dietético absorvido, incorporação, reabsorção e balanço de $\mathrm{P}$ no osso e nos tecidos moles e $\mathrm{P}$ proveniente do osso, dos tecidos moles e do total absorvido, que retorna ao trato gastrintestinal, em suínos em crescimento, alimentados com dietas contendo P de diferentes fontes de fosfato. O modelo usado foi determinístico e compartimental, em que o trato gastrintestinal (C1), os ossos (C2) e os tecidos moles, fígado, coração, rim e músculo, estudados em conjunto (C3), representaram os compartimentos. Foram utilizadas informações sobre metabolismo e cinética do fósforo em tecidos, obtidas pela técnica da diluição isotópica. Constatou-se que as hipóteses estabelecidas no modelo estão coerentes e os valores obtidos se ajustam ao fluxo do fósforo em suínos; as variáveis P consumido, P endógeno, que retorna ao trato gastrintestinal, $\mathrm{P}$ absorvido de origem dietética, retenção de $\mathrm{P}$, incorporação, reabsorção e balanço de $\mathrm{P}$ do osso e dos demais tecidos e fração de $\mathrm{P}$ do total absorvido que retorna ao trato gastrintestinal não foram influenciadas pelas fontes suplementares de fósforo; a origem do fósforo dietético pode interferir na distribuição do P nos tecidos, após o processo de absorção desse mineral; e o P proveniente do absorvido que retorna ao trato gastrintestinal foi proporcional ao $\mathrm{P}$ consumido.

Palavras-chave: absorção real, cinética, incorporação de fósforo, metabolismo mineral, perdas endógenas, radiofósforo

\section{Model of the Biological Flow of Phosphorus from Phosphate Sources in Pig, Using ${ }^{32} \mathbf{P}$ as Marker}

\begin{abstract}
The experiment was designed to simulate the mathematical models that relate to the phophorus (P) flow, considering the hypotheses that the endogenous phosphorus from digestive tract was absorbed in the same proportion of the dietary phosphorus and that the input and output flow of this mineral in the compartments was constant. The variables absorption, retention, endogenous $\mathrm{P}$ that returns to the gastrointestinal tract (GIT), dietary absorbed $\mathrm{P}$, accretion, reabsorption and balance of $\mathrm{P}$ in bone and in soft tissues and $\mathrm{P}$ from bone and soft tissue and from the total absorbed that returns to GIT, in growing pigs fed diets containing $\mathrm{P}$ from different phosphate sources were evaluate. The model was deterministic and compartmental, in which the GTI (C1), bones (C2) and soft tissues, liver heart, kidney and muscle, (C3) represented the compartments. The information on metabolism and kinetics of the P in tissues obtained by isotopic dilution technique was used. It was concluded that: the hypotheses established in the model were coherent and the obtained values were adjusted to the $\mathrm{P}$ flow in pigs; the variables $\mathrm{P}$ intake, endogenous $\mathrm{P}$ that return to the GIT, $\mathrm{P}$ retention, dietary absorbed $\mathrm{P}$, accretion, reabsorption and balance of $\mathrm{P}$ from bone and soft tissues and $\mathrm{P}$ from total absorbed that returned to the GIT were not influenced by the P from the phosphates sources; the dietary phosphorus could interfere on $\mathrm{P}$ distribution in the tissues after the process of absorption of that mineral; the $\mathrm{P}$ from total absorbed that return to the GIT was proportional to $\mathrm{P}$ intake.
\end{abstract}

Key Words: endogenous losses, kinetics, mineral metabolism, phosphorus accretion, radiophosphorus, true absorption

\section{Introdução}

A despeito das óbvias diferenças entre espécies, muitos critérios são usados para avaliar a utilização de fósforo nos alimentos, incluindo ganho de peso, eficiência alimentar, resistência à quebra do osso, cinza no osso, fósforo no soro sangüíneo, anormalidades no esqueleto, densidade do osso, distribuição do fósforo em tecidos, atividade da fosfatase alcalina, tamanho da leitegada, balanço metabólico e a taxa de incorporação e a biodisponibilidade, por meio da técnica da diluição isotópica (KLEIBER et al., 1951;

\footnotetext{
1 Pesquisa financiada pela FAPESP e parte do relatório de pós-doutorado do primeiro autor

2 Professor adjunto do Departamento de Zootecnia do CCA-UFPI, Campus Universitário da Socopo - Teresina - PI, 64049-550. E-mail:lopesjb@uol.com.br

3 Professor do Centro de Energia Nuclear na Agricultura da USP. E.mail: vitti@cena.usp.br; abdalla@cena.usp.br

4 Professora, Departamento de Entomologia - Escola Superior de Agricultura Luiz de Queiroz - USP.

5 Analista de Sistema, Departamento de Entomologia - Escola Superior de Agricultura Luiz de Queiroz - USP
} 
COMAR et al., 1953; AMMERMAN et al., 1963; PEELER, 1972; PROCHÁZKA et al., 1972; FISHER, 1978; BELLAVER et al., 1984; GOMES, 1988; VITTI, 1989; CARVALHO, 1998; e LOPES, 1998). Nesse sentido, a nutrição animal tem alcançado grandes avanços, com vários pesquisadores, em todo o mundo, preocupados em estudar os mais diferentes aspectos do metabolismo e da utilização racional dos diversos nutrientes essenciais ao organismo.

Assim, face à grande disponibilidade de informações e do conhecimento gerado nos amplos ramos da ciência, os cientistas têm se voltado a simular e desenvolver modelos matemáticos que possam se ajustar aos fenômenos biológicos. Tratam-se de modelos estocásticos, que envolvem componentes obtidos de forma casualizada, ou determinística, podendo, nas duas situações, representar descrições de grupos populacionais dentro de um espaço de tempo ou referir-se a processos dinâmicos (BROWN e ROTHERY, 1993).

Nesse contexto, a análise compartimental, em estudo de modelagem nos sistemas biológicos, tem sido bastante usada para sintetizar modelos mais simples, que simulam o comportamento relacionado ao metabolismo e à cinética de nutrientes no organismo animal (SAUVANT et al., 1996). A esse respeito, GRACE (1981) destaca que os compartimentos não podem ser definidos em termos anatômicos, porém estão relacionado com os processos metabólicos. No caso de avaliação do fósforo, o autor relata que é importante a hipótese estabelecida no modelo de que seja constante o fluxo de entrada e saída do mineral nos compartimentos. Assim, BASTIANELLI et al. (1996), estudando o modelo mecanístico da digestão de carboidratos, proteína, nitrogênio não-protéico, lipídeos e minerais em suínos, consideraram quatro compartimentos anatômicos, o estômago, o intestino delgado, dividido em duas partes, a primeira constituída de duodeno mais a parte proximal do jejuno e o restante do jejuno maisíleo e oquarto compartimento, ointestino grosso. Cada compartimento anatômico, por sua vez, foi dividoem subcompartimentos bioquímicos.

De acordo com KEBREAB e FRANCE (1999), um modelo biomatemático é caracterizado por uma equação ou um conjunto de equações representativas do comportamento de um sistema biológico, e seu uso tem se tornado parte integral das pesquisas nas mais distintas áreas de conhecimento. Segundo GARCIA (1997), simulação é a obtenção da resposta temporal das variáveis de interesse (dependentes) de um modelo, quando excita suas variáveis de entrada com sinais desejados e se definem valores das condições iniciais das variáveis dependentes.

SCHNEIDER et al. (1985) relatam que, apesar de a modelagem se tratar de uma técnica precisa, os autores mostram que, em estudos de absorção de nutrientes, devido ao modelo envolver medições instantâneas ou pontuais, é provável ocorrer diferenças dos resultados a partir da absorção baseada em observações de vários dias.

Objetivou-se com o presente trabalho testar hipóteses e simular modelos matemáticos relacionados ao fluxo de fósforo e avaliar absorção, retenção, $P$ endógeno que retorna ao trato gastrintestinal, incorporação, reabsorção e balanço de $\mathrm{P}$ no osso e nos tecidos e $\mathrm{P}$ proveniente do osso e tecidos que retorna ao trato gastrintestinal, para suínos em crescimento, alimentados com dietas, contendo $\mathrm{P}$ de diferentes fontes de fosfato (bicálcico, Tapira, Patos de Minas e monoamônio, superfosfato triplo e dieta controle, sem $\mathrm{P}$ suplementar).

Para elaboração do modelo, partiu-se das hipóteses de que o fósforo endógeno que chega ao trato digestivo é absorvido na mesma proporção do fósforo da dieta e o fluxo de entrada e saída do fósforo nos compartimentos é constante.

\section{Material e Métodos}

O modelo matemático do metabolismo e do fluxo de fósforo (Figura 1) foi adaptado dos estudos de FERNANDEZ (1995) e GRACE (1981). Foram utilizados resultados de pesquisa sobre metabolismo e cinética do fósforo, em tecidos de suínos em crescimento, por intermédio da técnica de diluição isotópica, usando-se, por via endovenosa (veia cava), 7,4 MBq $\mathrm{de}^{32} \mathrm{P}$ por leitão, como marcardor. Os animais foram alimentados de dietas (Tabela 1) com o $P$ proveniente dos fosfatos bicálcico (BIC), Tapira (TAP) e Patos de Minas (FPM), monoamônio (MAP), superfosfato triplo (SPT) e dieta controle (CONT), sem P suplementar (FIGUEIRÊDO, 1998).

$\mathrm{O}$ delineamento experimental foi o inteiramente ao acaso, com seis tratamentos (fontes) e quatro repetições para as variáveis de metabolismo e duas para as dos tecidos. Os resultados foram submetidos à analise de variância e a comparação das médias foi realizada pelo teste Duncan (STATISTICAL ANALYSIS SISTEM, 1986).

O modelo foi determinístico compartimental, em que o compartimento-1 (C1) representou o trato gastrintestinal; $\mathrm{C} 2$, os ossos; e C3, os tecidos moles, o 
Rev. bras. zootec.

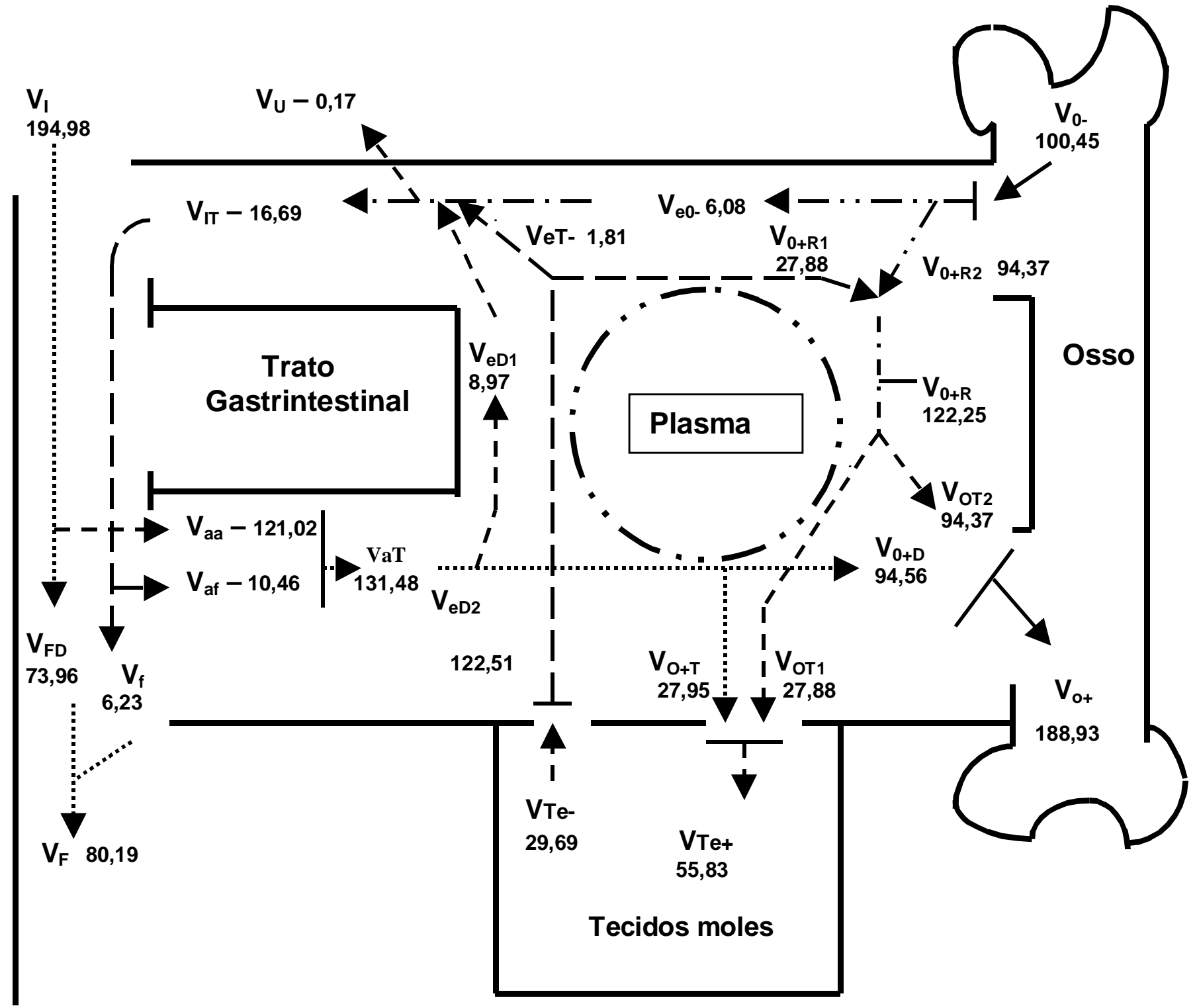

Figura 1 - Modelo da distribuição do P da dieta suplementada com fosfato Tapira, em suínos em crescimento, com o consumo diário de 214,15 mg P/kg PV .dia

Figure 1 - P distribution model of the diet phosphate supplemented with Tapira phosphate in growing pigs fed $214.15 \mathrm{mg} P / \mathrm{kg} L W . d a y$.

LEGENDA: VI - quantidade de $\mathrm{P}$ ingerido ( $P$ intake); $\mathrm{V}_{\mathrm{F}}$ - perda total de $\mathrm{P}$ nas fezes (total fecal $\mathrm{P}$ loss); $\mathrm{V}_{\mathrm{f}}$ - $\mathrm{P}$ endógeno fecal (endogenous fecal $P$ ); VFD - $P$ nas fezes de origem dietética (Fecal $P$ from dietary origin); $V_{U}$ - perda de $P$ na urina (urinary $P$ loss); $V_{a a}-P$ absorvido de origem alimentar (absorbed $P$ from dietary origin); $\mathrm{V}_{\mathrm{IT}} \mathrm{P}$ de origem endógena, que chega ao trato gastrintestinal - $\mathrm{T}_{\mathrm{Gl}}$ (Endogenous origin $P$ into GIT); $\mathrm{V}_{\mathrm{af}}-\mathrm{P}$ endógeno reabsorvido no TGI (endogenous reabsorbed $P$ in the GTI); $V_{a T}$ - total de $\mathrm{P}$ absorvido (total absorbed of $P$ ); $V_{0_{+}}-P$ incorporado no osso ( $P$ accretion in the bone); $\mathrm{V}_{\mathrm{O}_{-}}-\mathrm{P}$ reabsorvido do osso (Reabsorbed $\mathrm{P}$ in the bone); $\mathrm{V}_{\mathrm{O}_{2}}-\mathrm{P}$ do osso reciclado para o osso e tecidos moles $(P$ from bone recycled to bone and soft tissues); $\mathrm{V}_{\mathrm{eO}}$ - $\mathrm{P}$ reabsorvido do osso, que retorna ao TGl (reabsorbed $P$ from the bone into the GIT); $\mathrm{V}_{\mathrm{O}} \mathrm{D}-\mathrm{P}$ do $\mathrm{V}_{\mathrm{aT}}$ incorporado no osso ( $P$ from VaT accretion in the bone); $\mathrm{V}_{\mathrm{Te}+}-\mathrm{P}$ incorporado nos tecidos moles ( $P$ accretion in the soft tissues); $\mathrm{V}_{\mathrm{Te}-}-\mathrm{P}$ reabsorvido dos tecidos moles (Reabsorbed $P$ from the soft tissues); $V_{0}+P$ dos tecido moles reciclado para osso e tecidos moles ( $P$ from the soft tissue recycled into bone and soft tissues); $\mathrm{V}_{\mathrm{o}+\mathrm{R}}$ - total de $\mathrm{P}$ reciclado para o osso e tecidos moles (total $\mathrm{P}$ recycled into bone and soft tissues); $\mathrm{V}_{\mathrm{e}} \mathrm{T}$ - $\mathrm{P}$ reabsorvido dos tecidos moles, que retorna ao TGI (Reabsorbed $P$ from the soft tisssues into the GIT); $V_{O+T}-\mathrm{P}$ do $\mathrm{V}_{\mathrm{aT}}$ incorporado nos tecidos moles ( $P$ from VaT to accretion in the soft tissues); $\mathrm{V}_{0 \mathrm{D} 1}-\mathrm{P}$ do $\mathrm{V}_{\text {TT }}$ que retorna para o TGI (P from VaT into GIT); $\mathrm{V}_{\mathrm{e} 2}$ - $\mathrm{P}$ do $\mathrm{V}_{\mathrm{aT}}$ distribuído para o osso e tecidos moles ( $P$ from VaT into bone and soft tissues); $\mathrm{V}_{\mathrm{T} 01}-\mathrm{P}$ do $\mathrm{V}_{\mathrm{O}+\mathrm{R}}$ reincorporado nos tecidos moles ( $P$ from $V_{0+R}$ accretion into the soft tissues); $\mathrm{V}_{\mathrm{T} 02}-\mathrm{P}$ do $\mathrm{V}_{\mathrm{O}+\mathrm{R}}$ reincorporada no osso ( $P$ from $V_{0+R}$ accretion into bone). 
Tabela 1 - Composição das rações experimentais

Table 1 - Composition of the experimental diets

\begin{tabular}{|c|c|c|c|c|c|c|}
\hline $\begin{array}{l}\text { Ingrediente }(\mathrm{kg}) \\
\text { Ingredient }\end{array}$ & BIC & TAP & FPM & MAP & SPT & CONT \\
\hline Milho & 71,50 & 71,20 & 70,64 & 69,72 & 70,54 & 72,16 \\
\hline $\begin{array}{l}\text { Corn } \\
\text { Farelo de soja }\end{array}$ & 24,00 & 24,00 & 24,20 & 24,30 & 24,20 & 24,00 \\
\hline $\begin{array}{l}\text { Soybean meal } \\
\text { Óleo de soja } \\
\text { Soybean oil }\end{array}$ & 1,20 & 1,27 & 1,50 & 1,80 & 1,50 & 0,94 \\
\hline $\begin{array}{l}\text { Calcário } \\
\text { Limestone }\end{array}$ & 1,00 & 0,53 & 0,06 & 2,00 & 1,46 & 2,00 \\
\hline $\begin{array}{l}\text { Fosfato bicálcico } \\
\text { Dicalcium phosphate }\end{array}$ & 1,40 & - & - & - & - & - \\
\hline $\begin{array}{l}\text { Fosfato Tapira } \\
\text { Tapira phosphate }\end{array}$ & - & 2,10 & - & - & - & - \\
\hline $\begin{array}{l}\text { Fosfato Patos Minas } \\
\text { Patos de Minas phosphate }\end{array}$ & - & - & 2,70 & - & - & - \\
\hline $\begin{array}{l}\text { Fosfato monoamônio } \\
\text { Monoammonium phosphate }\end{array}$ & - & - & & 1,28 & - & - \\
\hline $\begin{array}{l}\text { Fosfato supertriplo } \\
\text { Triple superphosphate }\end{array}$ & - & - & - & - & 1,40 & - \\
\hline $\begin{array}{l}\text { Sal } \\
\text { Salt }\end{array}$ & 0,40 & 0,40 & 0,40 & 0,40 & 0,40 & 0,40 \\
\hline $\begin{array}{l}\text { Suplemento mineral }{ }^{1} \\
\text { Mineral supplement }^{1} \\
\text { Suplemento vitamínico } \\
\text { Vitamin supplement }^{2}\end{array}$ & 0,10 & 0,10 & 0,10 & 0,10 & 0,10 & $\begin{array}{l}0,10 \\
0,40\end{array}$ \\
\hline & \multicolumn{6}{|c|}{ Composição analisada (Analyzed composition) } \\
\hline $\begin{array}{l}\mathrm{PB} \\
C P\end{array}$ & 15,41 & 14,94 & 14,58 & 15,20 & 14,36 & 14,79 \\
\hline $\begin{array}{l}\mathrm{ED}^{3}(\mathrm{kcal} / \mathrm{kg}) \\
D E^{3}(\mathrm{kcal} / \mathrm{kg})\end{array}$ & 3404 & 3399 & 3403 & 3399 & 3399 & 3405 \\
\hline $\begin{array}{l}\text { Fósforo total } \\
\text { Total phosphorus }\end{array}$ & 0,56 & 0,56 & 0,56 & 0,56 & 0,56 & 0,30 \\
\hline $\begin{array}{l}\text { Cálcio } \\
\text { Calcium }\end{array}$ & 0,75 & 0,75 & 0,75 & 0,75 & 0,75 & 0,75 \\
\hline $\begin{array}{l}\text { Flúor (ppm) } \\
\text { Fluoride (ppm) }\end{array}$ & 12,32 & 203,07 & 467,10 & 65,28 & 54,60 & - \\
\hline
\end{tabular}

1 Quantidade/kg de dieta (Amount/kg of diet): Fe, 80 mg; Cu, 12 mg; Mn, 70 mg; Zn, 100 mg; I, 1 mg; Se, $0,12$.

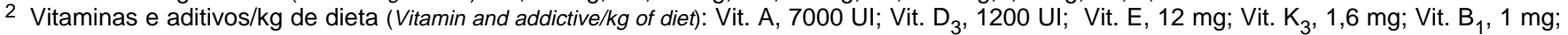
Vit. $\mathrm{B}_{2}, 3 \mathrm{mg}$; Vit $\mathrm{B}_{6}, 1 \mathrm{mg}$; Vit $\mathrm{B}_{12}, 12 \mathrm{mg}$; niacina (niacin), 20 mg; ácido pantotênico (pantothenic acid), $12 \mathrm{mg}$; Colina (choline), $150 \mathrm{mg}$; Promotor crescimento (growth promoter) $48 \mathrm{mg}$; Antioxidante (antioxidant) $15 \mathrm{mg}$.

3 Baseado em ROSTAGNO et al. (1987) (Based on ROSTAGNO et al., 1987).

fígado, coração, rim e músculo, estudados em conjunto, e observando-se a proporção dos seus pesos. As variáveis analisadas foram relacionadas ao $\mathrm{P}$ consumido.

Para o estudo do metabolismo de $\mathrm{P}$, as equações envolvendo as variáveis $\mathrm{P}$ consumido, $\mathrm{P}$ total excretado nas fezes $\left(\mathrm{V}_{\mathrm{F}}\right)$, com as frações de origem alimentar $\left(\mathrm{V}_{\mathrm{FD}}\right)$ e endógena $\left(\mathrm{V}_{\mathrm{f}}\right)$, $\mathrm{P}$ excretado na urina $\left(\mathrm{V}_{\mathrm{U}}\right)$, absorção de $\mathrm{P}$ de origem alimentar $\left(\mathrm{V}_{\mathrm{aa}}\right)$ e reabsorção do $P$ de origem endógena (Vaf), valor da fração de $P$ endógeno, proveniente de secreções e células de descamação, que chega ao trato gastrintestinal $\left(\mathrm{V}_{\mathrm{IT}}\right)$, absorção total de $\mathrm{P}$, incluindo as frações de origem endógena e alimentar $\left(\mathrm{V}_{\mathrm{aT}}\right)$ e balanço do $\mathrm{P}(\mathrm{Ba})$ foram definidas pelas Equações 1 - 6, segundo FERNANDEZ (1995). O valor de P proveniente do total absorvido (VaT) que retorna ao trato grastrintestinal $\left(\mathrm{V}_{\mathrm{eD1}}\right)$ foi calculado pela diferença entre o P do VaT e o P incorporado em C2 e C3, após subtrair-se o $\mathrm{P}$ reciclado que retorna a esses compartimentos. $\mathrm{O}$ restante do $\mathrm{P}$ total absorvido $\left(\mathrm{V}_{\mathrm{eD} 2}\right)$ foi distribuído proporcionalmente entre $\mathrm{C} 2 \mathrm{e} \mathrm{C} 3\left(\mathrm{~V}_{0+\mathrm{D}} \mathrm{e}\right.$ $\mathrm{V}_{0+\mathrm{T}}$ ), de acordo com as Equações 7 - 10. 
Rev. bras. zootec.

$\mathrm{V}_{\mathrm{FD}}=\mathrm{V}_{\mathrm{I}}-\mathrm{V}_{\mathrm{f}}$

$\mathrm{V}_{\mathrm{aa}}=\mathrm{V}_{\mathrm{I}}-\mathrm{V}_{\mathrm{FD}}$

$\mathrm{V}_{\mathrm{IT}}=\mathrm{V}_{\mathrm{f}} /\left[1-\left(\mathrm{V}_{\mathrm{aa}} / \mathrm{V}_{\mathrm{f}}\right)\right]$

$\mathrm{V}_{\mathrm{af}}=\mathrm{V}_{\mathrm{IT}}-\mathrm{V}_{\mathrm{f}}$

$\mathrm{V}_{\mathrm{aT}}=\mathrm{V}_{\mathrm{aa}}-\mathrm{V}_{\mathrm{af}}$

$\mathrm{Ba}=\mathrm{V}_{\mathrm{I}}-\mathrm{V}_{\mathrm{F}}-\mathrm{V}_{\mathrm{U}}$

$\mathrm{V}_{\mathrm{eD} 1}=\mathrm{V}_{\mathrm{aT}}-\left(\mathrm{V}_{0}+-\mathrm{V}_{0}+{ }_{\mathrm{R} 2}\right)-\left(\mathrm{V}_{\mathrm{Te}}+-\mathrm{V}_{0}{ }_{\mathrm{R} 1}\right)$

$\mathrm{V}_{\mathrm{eD} 2}=\mathrm{V}_{\mathrm{aT}}-\mathrm{V}_{\mathrm{eD} 1}$

$\left[\mathrm{V}_{0}+\mathrm{x}\left(\mathrm{V}_{\mathrm{aT}} \mathrm{x} \% \mathrm{P}\right.\right.$ incorporado no osso $\left.)\right]$

$\mathrm{V}_{\mathrm{o}+\mathrm{D}}=\frac{}{\left[\mathrm{V}_{0}-\mathrm{x}\left(\mathrm{V}_{\mathrm{aT}} \mathrm{x} \% \mathrm{P} \text { incorporado no osso }\right)\right]}$

(Equação 9)

$\mathrm{V}_{\mathrm{O}+\mathrm{T}}=\frac{\left[\mathrm{V}_{\mathrm{Te}}+\mathrm{x}\left(\mathrm{V}_{\mathrm{aT}} \mathrm{x} \% \text { P incorporado nos tecidos }\right)\right]}{\left[\mathrm{V}_{\mathrm{Te}-} \mathrm{x}\left(\mathrm{V}_{\mathrm{aT}} \mathrm{x} \% \mathrm{P} \text { incorporado nos tecidos }\right)\right]}$ (Equação 10)

$\mathrm{Na}$ determinação do $\mathrm{P}$ incorporado no osso $\left(\mathrm{V}_{0_{+}}\right)$e nos tecidos moles, fígado, coração, rim e músculo $\left(\mathrm{V}_{\mathrm{Te}+}\right)$, consideraram-se a concentração de P (mg/g MS), o percentual de ${ }^{32} \mathrm{P}$ [(cpm amostra/g de MS)/(dose total injetada)]/[(mg P/g MS)], e a atividade específica dos tecidos[(\% de atividade do ${ }^{32} \mathrm{P}$ injetada)/(mg P/g MS do tecido)], segundo SMITH et al. (1951), VITTI et al. (1992) e LOPES (1998). Com relação ao cálculo do ${ }^{32} \mathrm{P}$ retido nos tecidos moles, foi considerada a média dos valores encontrados em cada tecido estudado. Já a quantidade de matéria seca (MS) de cada tecido foi determinada pelo produto entre o percentual de MS obtido e o peso dos tecidos, definido segundo dados de pesquisa de DAVIDSON (1966) e LOPES (1988). Foi utilizado, ainda, nesse processo, o decaimento físico (T1/2) e biológico (Tb) do ${ }^{32} \mathrm{P}$, fundamentado na INTERNATIONAL ATOMIC ENERGY AGENCY (1979), uma vez que os animais foram sacrificados uma semana após a introdução do ${ }^{32} \mathrm{P}$ e a contagem do material radioativo 14 dias após o abate.

Nesse contexto, o valor do ${ }^{32} \mathrm{P}$ retido (mg/g MS) foi obtido pelo produto da concentração de $\mathrm{P}$ total nos compartimentos $\mathrm{C} 1$ e $\mathrm{C} 2$ (mg/g MS) e o respectivo $\mathrm{P}$ retido (\%). Após a correção do decaimento do ${ }^{32} \mathrm{P}$ para a data de sua introdução na corrente sangüínea, calculou-se o P incorporado nos compartimentos-2 e 3 (mg/g MS) pela relação entre o valor de ${ }^{32} \mathrm{P}$ corrigido e o valor da atividade específica em cada compartimento envolvido, e o $\mathrm{P}$ total incorporado $(\mathrm{mg}$ ) pelo produto entre a MS ( $\mathrm{g}$ ) do compartimento e o P incorporado (mg/g MS). Os valores de $\mathrm{V}_{0+}$ e $\mathrm{V}_{\mathrm{Te}+}$ (mg P/kg PV.dia) foram obtidos pela relação entre o $P$ total incorporado no compartimento e o peso do animal (g). As Equações 11-24 caracterizam essas determinações.
${ }^{32}$ Pretido no osso $\left(\mathrm{mg}{ }^{32} \mathrm{P} / \mathrm{g} \mathrm{MS}\right)=\mathrm{P}$ inorgânico $(\mathrm{mg} \mathrm{P} / \mathrm{g} \mathrm{MS}) \times{ }^{32} \mathrm{P}$ retido $(\%)$ (Equação 11)

$\mathrm{P}$ total incorporado osso $(\mathrm{mg} \mathrm{P})=\mathrm{P}$ total osso $(\mathrm{mg} \mathrm{P} / \mathrm{g}$ MS) $\mathrm{x}$ MS osso $(\mathrm{g} \mathrm{MS})$ (Equação 12)

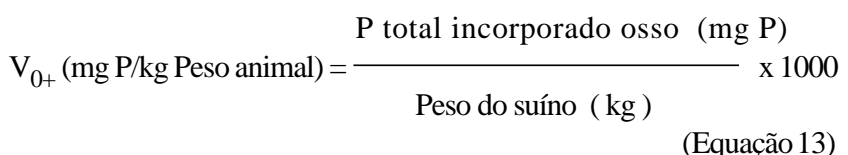

Peso total tecidos $(\mathrm{g})=$ Peso suíno $(\mathrm{g})$ x \% Peso tecidos (Equação 14)

MS tecidos $(\mathrm{g})=$ Peso total tecidos $(\mathrm{g})$ x \% MS tecidos (Equação 15)

${ }^{32} \mathrm{P}$ retido fígado $(\mathrm{mg}$ 32 $\mathrm{P} / \mathrm{g} \mathrm{MS})=\mathrm{P}$ inorgânico $(\mathrm{mg} \mathrm{P} / \mathrm{g} \mathrm{MS}) \mathrm{x}{ }^{32} \mathrm{P}$ retido fígado (\%) (Equação 16)

${ }^{32} \mathrm{P}$ retido coração (mg ${ }^{32} \mathrm{P} / \mathrm{g}$ MS ) = P inorgânico (mg P/g MS) $\mathrm{x}{ }^{32} \mathrm{P}$ retido (\%) (Equação 17)

${ }^{32}$ Pretidorim $\left(\mathrm{mg}{ }^{32} \mathrm{P} / \mathrm{gMS}\right)=$ Pinorgânico $(\mathrm{mgP} / \mathrm{gMS}) \mathrm{x}{ }^{32} \mathrm{Pretido}(\%)$ (Equação 18)

${ }^{32} \mathrm{P}$ retido músculo $\left(\mathrm{mg}{ }^{32} \mathrm{P} / \mathrm{g} \mathrm{MS}\right)=\mathrm{P}$ inorgânico $(\mathrm{mg} \mathrm{P} / \mathrm{g} \mathrm{MS}) \mathrm{x}{ }^{32} \mathrm{P}$ retido (\%) (Equação 19)

$\mathrm{P}$ total incorporado tecido $(\mathrm{mg} \mathrm{P})=\mathrm{P}$ total tecido $(\mathrm{mg} \mathrm{P} / \mathrm{g} \mathrm{MS}) \times$ MS tecido $(\mathrm{g})$ (Equação 20)

$\mathrm{V}_{\mathrm{Te}+}(\mathrm{mg} \mathrm{P} / \mathrm{kg}$ peso animal $)=\stackrel{1}{\longrightarrow} 1000$ Peso do suíno ( $\mathrm{kg}$ )

(Equação 21)

$\mathrm{T} 1 / 2=0,693 /$ constante de decaimento $(\mathrm{K}) \quad$ (Equação 22)

Atividade do material radioativo no tempo "t" (A) = Atividade inicial $\left(\mathrm{A}_{0}\right) \mathrm{x} \mathrm{e}^{-\mathrm{kt}}$

(Equação 23)

$\mathrm{Tb}=0,693 \mathrm{t} / \ln \left(\mathrm{A}_{0} / \mathrm{A}\right)$

(Equação 24)

$\mathrm{O} P$ reabsorvido nos compartimentos $\mathrm{C} 2\left(\mathrm{~V}_{0_{-}}\right) \mathrm{e}$ $\mathrm{C} 3\left(\mathrm{~V}_{\mathrm{Te}}\right)$ foi calculado deduzindo-se, respectivamente, de $\mathrm{V}_{0+}$ e $\mathrm{V}_{\mathrm{Te}+} \mathrm{O}$ valor do balanço de $\mathrm{P}$ multiplicado pela proporção de $\mathrm{P}$ incorporado no compartimento em estudo (Equações 25 e 26). Para determinação do P reciclado que retorna aos compartimentos $\mathrm{C} 2$ e $\mathrm{C} 3\left(\mathrm{~V}_{0+\mathrm{R} 1}\right.$ e $\left.\mathrm{V}_{0+\mathrm{R} 2}\right)$ e ao trato gastrointestinal ( $\mathrm{V}_{\mathrm{eo}-} \mathrm{e}_{\mathrm{eT}_{-}}$), o cálculo foi realizado com base nas equações 27-30, segundo FERNANDEZ (1995), adaptando-se ao modelo a proporção incorporada em cada um dos dois compartimentos, visto que neste trabalho o osso foi considerado como único compartimento, que mobilizou P. Ovalor do P proveniente do osso e tecidos moles reciclado para os tecidos moles $\left(\mathrm{V}_{0+\mathrm{R}}\right)$, o do $\mathrm{P}$ do $\mathrm{V}_{0+\mathrm{R}}$ reincorporado nos tecidos 
moles $\left(\mathrm{V}_{\mathrm{T} 01}\right)$ e o $\mathrm{P}$ do $\mathrm{V}_{0+\mathrm{R}}$ reincorporado nos ossos $\left(\mathrm{V}_{\mathrm{T} 02}\right)$ foram determinados de acordo com as Equações 31-33.

$\mathrm{V}_{0-}=\mathrm{V}_{0+}-(\mathrm{Ba} \times \% \mathrm{P}$ incorporado osso) $\quad$ (Equação 25)

$\mathrm{V}_{\mathrm{Te}-}=\mathrm{V}_{\mathrm{Te}+}-(\mathrm{Ba} \times \mathrm{P}$ incorporado tecido) (Equação 26)

$\mathrm{V}_{0+\mathrm{R} 1}=\frac{\mathrm{V}_{\mathrm{Te}+} \times \mathrm{V}_{\mathrm{Te}-}}{\left[\mathrm{V}_{\mathrm{Te}+}\left(\mathrm{V}_{\mathrm{aT}} \times \% \text { P incorporado tecido }\right)\right]}$ (Equação 27)

$\mathrm{V}_{0+\mathrm{R} 2}=\frac{\mathrm{V}_{0+} \times \mathrm{V}_{0-}}{\mathrm{V}_{0-}+\left(\mathrm{V}_{\mathrm{aT}} \times \% \mathrm{P} \text { incorporado osso }\right)}$ (Equação 28)

$\mathrm{V}_{\mathrm{e} 0-}=\mathrm{V}_{0-}-\mathrm{V}_{0+\mathrm{R} 2}$

$\mathrm{V}_{\mathrm{eT}-}=\mathrm{V}_{\mathrm{Te}-}-\mathrm{V}_{0+\mathrm{R} 1}$

$\mathrm{V}_{0+\mathrm{R}}=\mathrm{V}_{0+\mathrm{R} 1}+\mathrm{V}_{0+\mathrm{R} 2}$

$\mathrm{V}_{\mathrm{T} 01}=\mathrm{V}_{0+\mathrm{R}}-\left(\mathrm{V}_{0+}-\mathrm{V}_{0+\mathrm{D}}\right)$

$\mathrm{V}_{\mathrm{T} 02}=\mathrm{V}_{0+\mathrm{R}}-\mathrm{V}_{\mathrm{T} 01}$

(Equação 29)

(Equação 30)

(Equação 31)

(Equação 32)

(Equação 33)

\section{Resultados e Discussão}

Os valores obtidos de acordo com o modelo utilizado encontram-se na Tabela 2.

As variáveis $\mathrm{P}$ consumido, $\mathrm{P}$ de origem endógena que retorna ao trato gastrintestinal, $\mathrm{P}$ urinário, $\mathrm{P}$ total das fezes e as frações de origem endógena e dietética, $\mathrm{P}$ absorvido de origem dietética, retenção de $\mathrm{P}$, disponibilidade biológica de $\mathrm{P}$ das fontes de fosfato, incorporação, reabsorção e balanço de $\mathrm{P}$ do osso e dos demais tecidos, e $\mathrm{P}$ do VaT que retorna ao trato digestivo não foram influenciados pela ingestão de $P$ suplementar das fontes de fosfato bicálcico, Tapira, Patos de Minas, monoamônio e supertriplo $(\mathrm{P}>0,05)$. Esse comportamento ressalta a importância dessas fontes estudadas como suplemento de P, em dietas de suínos em crescimento, e está em consonância com as observações de FIGUEIRÊDO (1998), usando os mesmos animais deste estudo, ao constarem que essas fontes não interferiram no consumo e na excreção de $\mathrm{P}$ nas fezes e na urina, na fração endógena fecal, na taxa de absorção e concentração de $\mathrm{P}$ no plasma. Esta constatação sugere que as hipóteses estabelecidas no modelo estão coerentes e os valores obtidos para as variáveis estudadas se ajustam ao fluxo do fósforo no organismo de suínos em crescimento.

Como a disponibilidade biológica do fósforo das diferentes fontes de fosfatos não diferiram entre si, fica caracterizada a similaridade na eficiência da utilização do fósforo desses fosfatos pelos suínos em crescimento. Nesse contexto, acredita-se que, mesmo considerando o curto período experimental, o conteúdo de flúor nos fosfatos estudados não interferiu no aproveitamento do fósforo pelo leitões, credenciando essas fontes como importante alternativa de fósforo suplementar para suínos. A esse respeito, GOMES et al. (1985) e BARBOSA et al. (1992), ao desenvolverem vários trabalhos de pesquisa com fosfatos de rocha, na alimentação de suínos em crescimento e terminação, constataram que o flúor não provocou efeito prejudicial sobre o desempenho dos animais.

A ordem decrescente, encontrada nos valores da disponibilidade de fósforo dos fosfatos estudados no presente trabalho, foi monoamônio (85,63\%), bicálcico $(84,76 \%)$, supertriplo $(75,83 \%)$, Tapira $(73,03 \%)$ e Patos de Minas $(70,86 \%)$, sendo similar à ordem da disponibilidade relativa observada nas pesquisas de FURTADO (1991) GOMES et al. (1992).

Os resultados de $\mathrm{P}$ incorporado no osso foram similares $(\mathrm{P}>0,05)$ aos de FERNANDEZ (1995); os do $\mathrm{P}$ reabsorvido, superiores $(\mathrm{P}<0,05)$; e para as demais variáveis estudadas, inferiores $(\mathrm{P}<0,05)$. Estes valores inferiores podem estar relacionados ao menor consumo de $\mathrm{P}$ pelos animais deste experimento e além disso, o consumo desse mineral foi inferior ao recomendado pelo NATIONAL RESEARCH COUNCIL - NRC (1998). Também, podem ter contribuído nesse processo as prováveis diferenças nas condições nutricionais dos animais no início da pesquisa e o curto período experimental de 14 dias. Nesse sentido, ENGSTRON et al. (1985) ressaltam que a hipofosfatemia se manifesta progressivamente em suínos submetidos a dietas deficientes em fósforo, atingindo a média mínima de $\mathrm{P}$ inorgânico no plasma, em torno de 34 a 41 dias de restrição.

Já os dados de $\mathrm{P}$ endógeno de origem dietética, retenção e total de $\mathrm{P}$ absorvido, balanço de $\mathrm{P}$ no osso e $\mathrm{P}$ reabsorvido dos tecidos foram similares aos encontrados por LOPES et al. (1999), em pesquisa com suínos em crescimento, com consumo de P similar.

A fração de $\mathrm{P}$ absorvido de origem endógena da dieta com fosfato monoamônio foi semelhante à do fosfato bicálcico $(\mathrm{P}>0,05)$ e superior ao do Tapira, Patos de Minas e supertriplo $(\mathrm{P}<0,05)$ e o do bicálcico, semelhante ao Tapira e ao supertriplo $(P>0,05)$. Essa ocorrência pode estar ligada ao fato de o P total absorvido do fosfato monoanônio ter apresentado maior valor absoluto, o que pode ter interferido no maior valor do $\mathrm{P}$ endógeno reabsorvido, visto que, no desenvolvimento do modelo, foi estabelecida a hipótese 
Rev. bras. zootec.

Tabela 2 - Médias das variáveis relacionadas com o estudo e do metabolismo e cinética do fósforo de diferentes fontes em suínos em crescimento

Table 2 - Means of the variables related to the metabolism and kinetic of phosphorus from different phosphates sources in growing pigs

\begin{tabular}{|c|c|c|c|c|c|c|c|}
\hline $\begin{array}{l}\text { Parâmetro }^{1} \text { (mg/kg PV.dia) } \\
\text { Parameter }^{1} \text { (mg/kg LW. day) }\end{array}$ & BIC & TAP & FPM & MAP & SPT & CONT & EPM $^{1}$ \\
\hline $\begin{array}{l}\mathrm{P} \text { consumido } \\
\text { Intake } P\end{array}$ & $194,98^{\mathrm{a}}$ & $214,15^{a}$ & $224,58^{a}$ & $230,20^{\mathrm{a}}$ & $201,35^{\mathrm{a}}$ & $92,79^{b}$ & 15,17 \\
\hline $\begin{array}{l}\mathrm{P} \text { endógeno para TGI }\left(\mathrm{V}_{\mathrm{IT}}\right) \\
\text { Endogenous } P \text { into GIT }\end{array}$ & $16,69^{\mathrm{a}}$ & $10,57^{\mathrm{a}}$ & $9,99^{a}$ & $16,72^{\mathrm{a}}$ & $9,85^{\mathrm{a}}$ & $13,59^{\mathrm{a}}$ & 2,66 \\
\hline $\begin{array}{l}\mathrm{P} \text { total fezes }\left(\mathrm{V}_{\mathrm{F}}\right) \\
\text { Fecal total } P\end{array}$ & $80,19^{a b}$ & $97,50^{\mathrm{ab}}$ & $109,34^{\mathrm{a}}$ & $81,99^{a b}$ & $107,98^{a}$ & $60,81^{b}$ & 14,32 \\
\hline $\begin{array}{l}\mathrm{P} \text { endógeno fecal }\left(\mathrm{V}_{\mathrm{f}}\right) \\
\text { Endogenous fecal } P\end{array}$ & $6,23^{\mathrm{a}}$ & $4,59^{a}$ & $4,72^{\mathrm{a}}$ & $5,73^{\mathrm{a}}$ & $5,05^{\mathrm{a}}$ & $7,89^{\mathrm{a}}$ & 1,78 \\
\hline $\begin{array}{l}\mathrm{P} \text { fecal origem alimentar }\left(\mathrm{V}_{\mathrm{FD}}\right) \\
\text { Fecal } P \text { from dietary origin }\end{array}$ & $73,97^{\mathrm{ab}}$ & $93,02^{\mathrm{a}}$ & $104,62^{a}$ & $76,27^{\mathrm{ab}}$ & $102,93^{\mathrm{a}}$ & $52,92^{b}$ & 12,95 \\
\hline $\begin{array}{l}\text { Purina }\left(\mathrm{V}_{\mathrm{U}}\right) \\
\text { Urinary } P\end{array}$ & $0,17^{\mathrm{a}}$ & $0,40^{\mathrm{a}}$ & $0,34^{\mathrm{a}}$ & $0,49^{\mathrm{a}}$ & $0,10^{\mathrm{a}}$ & $0,43^{\mathrm{a}}$ & 0,18 \\
\hline $\begin{array}{l}\mathrm{P} \text { dietético absorvido }\left(\mathrm{V}_{\mathrm{aa}}\right) \\
\text { Dietary absorbed } P\end{array}$ & $121,02^{\mathrm{ab}}$ & $121,13^{\mathrm{ab}}$ & $119,96^{\mathrm{ab}}$ & $153,93^{\mathrm{a}}$ & $98,42^{a b}$ & $39,88^{b}$ & 21,37 \\
\hline $\begin{array}{l}\mathrm{P} \text { endógeno absorvido }\left(\mathrm{V}_{\mathrm{af}}\right) \\
\text { Endogenous absorbed } P\end{array}$ & $10,46^{\mathrm{ab}}$ & $5,96^{\mathrm{bc}}$ & $5,27^{\mathrm{c}}$ & $11,00^{\mathrm{a}}$ & $4,80^{\mathrm{c}}$ & $5,70^{\mathrm{bc}}$ & 1,89 \\
\hline $\begin{array}{l}\mathrm{P} \text { total absorvido }\left(\mathrm{V}_{\mathrm{aT}}\right) \\
\text { Absorbed total } P\end{array}$ & $131,48^{a b}$ & $127,09^{\mathrm{ab}}$ & $125,23^{\mathrm{ab}}$ & $164,93^{\mathrm{a}}$ & $103,22^{b}$ & $45,58^{c}$ & 22,58 \\
\hline $\begin{array}{l}\text { Balanço de } \mathrm{P} \\
\text { P balance }\end{array}$ & $114,62^{\mathrm{ab}}$ & $116,12^{\mathrm{ab}}$ & $114,89^{\mathrm{ab}}$ & $147,72^{\mathrm{a}}$ & $93,26^{\mathrm{ab}}$ & $31,56^{\mathrm{b}}$ & 22,59 \\
\hline $\begin{array}{l}\text { Disponibilidade biológica } \\
\text { Biological availability }\end{array}$ & $84,76^{\mathrm{a}}$ & $73,03^{a}$ & $70,86^{\mathrm{a}}$ & $85,63^{\mathrm{a}}$ & $75,83^{\mathrm{a}}$ & - & 16,81 \\
\hline $\begin{array}{l}\mathrm{P} \text { incorporado osso }\left(\mathrm{V}_{\mathrm{o}+}\right) \\
P \text { accretion in bone }\end{array}$ & $188,93^{a}$ & $130,32^{\mathrm{a}}$ & $135,32^{\mathrm{a}}$ & $200,67^{a}$ & $167,46^{\mathrm{a}}$ & $148,07^{\mathrm{a}}$ & 28,72 \\
\hline $\begin{array}{l}\mathrm{P} \text { reabsorvido do osso }\left(\mathrm{V}_{\mathrm{o}}\right) \\
\text { Reabsorbed } P \text { in the bone }\end{array}$ & $100,45^{\mathrm{ab}}$ & $37,96^{\mathrm{b}}$ & $45,67^{b}$ & $81,76^{\mathrm{ab}}$ & $91,27^{\mathrm{ab}}$ & $123,52^{\mathrm{a}}$ & 27,02 \\
\hline $\begin{array}{l}\text { Balanço de } \mathrm{P} \text { no osso } \\
\text { P balance in the bone }\end{array}$ & $88,48^{\mathrm{ab}}$ & $92,36^{\mathrm{ab}}$ & $89,65^{\mathrm{ab}}$ & $118,91^{\mathrm{a}}$ & $76,19^{\mathrm{ab}}$ & $24,55^{\mathrm{b}}$ & 17,70 \\
\hline $\begin{array}{l}\mathrm{V}_{\mathrm{o}-} \text { para TGI e urina }\left(\mathrm{V}_{\mathrm{eo}-}\right) \\
V_{o-} \text { into GTI and urine }\end{array}$ & $6,08^{b}$ & $2,38^{\mathrm{c}}$ & $2,55^{\mathrm{c}}$ & $5,09^{\mathrm{b}}$ & $4,17^{b c}$ & $8,42^{\mathrm{a}}$ & 0,89 \\
\hline $\begin{array}{l}\mathrm{P} \text { para tecido moles }\left(\mathrm{V}_{\mathrm{Te}+}\right) \\
P \text { into soft tissues }\end{array}$ & $55,83^{\mathrm{a}}$ & $33,64^{\mathrm{a}}$ & $38,14^{\mathrm{a}}$ & $49,31^{\mathrm{a}}$ & $37,42^{\mathrm{a}}$ & $44,00^{\mathrm{a}}$ & 10,35 \\
\hline $\begin{array}{l}\mathrm{P} \text { reabsorvido tecidos moles }\left(\mathrm{V}_{\mathrm{Te}}\right) \\
\text { Reabsorbed } P \text { in the soft tissues }\end{array}$ & $29,69^{\mathrm{ab}}$ & $9,88^{\mathrm{b}}$ & $12,90^{\mathrm{b}}$ & $20,50^{\mathrm{ab}}$ & $20,35^{\mathrm{ab}}$ & $36,99^{\mathrm{a}}$ & 8,14 \\
\hline $\begin{array}{l}\text { Balanço P tecidos moles } \\
\text { P balance in the soft tissues }\end{array}$ & $26,14^{\mathrm{a}}$ & $23,76^{\mathrm{a}}$ & $25,24^{\mathrm{a}}$ & $28,81^{\mathrm{a}}$ & $17,07^{\mathrm{ab}}$ & $7,01^{\mathrm{b}}$ & 6,60 \\
\hline $\begin{array}{l}\mathrm{V}_{\mathrm{Te}} \text { para TGI e urina }\left(\mathrm{V}_{\mathrm{eT}-}\right) \\
V_{\text {Te- }} \text { into GIT and urine }\end{array}$ & $1,81^{\mathrm{ab}}$ & $0,62^{\mathrm{c}}$ & $0,75^{\mathrm{bc}}$ & $1,20^{\mathrm{bc}}$ & $0,97^{\mathrm{bc}}$ & $2,55^{\mathrm{a}}$ & 0,43 \\
\hline $\begin{array}{l}\mathrm{P} \text { do } \mathrm{V}_{\mathrm{aT}} \text { para o TGI }\left(\mathrm{V}_{\mathrm{eD} 1}\right) \\
P \text { from } V_{a T} \text { into } G I T\end{array}$ & $8,97^{\mathrm{ab}}$ & $7,97^{\mathrm{ab}}$ & $7,04^{\mathrm{ab}}$ & $10,92^{\mathrm{a}}$ & $4,82^{\mathrm{ab}}$ & $3,05^{\mathrm{b}}$ & 2,77 \\
\hline
\end{tabular}

${ }^{1}$ Médias seguidas de letras diferentes na mesma linha diferem $(P<0,05)$ pelo teste Duncan.

Means, within a row, followed by different letters are different $(P<.05)$ by Duncan test.

2 Erro-padrão da média (Standard error of mean).

de que o P endógeno total que chega ao trato digestivo é absorvido na mesma proporção do $\mathrm{P}$ dietético.

O P total absorvido do fosfato monoamônio foi semelhante ao do bicálcico, Tapira, Patos de Minas $(\mathrm{P}>0,05)$ e superior ao supertriplo e à dieta controle, que apresentou o menor valor entre os tratamentos estudados, devido, também, ao fato de os animais desse tratamento terem consumido menor quantidade de P. Além disso, a dieta controle não teve suplementação desse mineral e o P existente, por ser de origem vegetal, apresenta menor taxa de absorção, pois, segundo KETAREN et al. (1993), o fósforo fítico apresenta baixa disponibilidade biológica para leitões.

$\mathrm{O} \mathrm{P}$ reabsorvido do osso que retorna ao trato gastrintetinal $\left(\mathrm{V}_{\mathrm{eo}-}\right)$ da dieta controle, em que os animais consumiram menor quantidade de $\mathrm{P}$, foi superior ao das dietas suplementadas com fontes de $\mathrm{P}$ e o do fosfato bicálcico, semelhante ao do 
monoamônio e supertriplo e superior ao Tapira e Patos de Minas. Para estes últimos fosfatos, os valores foram semelhantes entre si e também ao supertriplo $((\mathrm{P}>0,05)$.

Como o $\mathrm{P}$ total absorvido das fontes monoamônio, bicálcico, Tapira e Patos de Minas foram semelhantes entre si e o valor do $\mathrm{V}_{\text {eo- }}$ do monoamônio e do bicálcico foi superior ao do Tapira e do Patos de Minas, este fato sugere que a distribuição do P nos tecidos, após o processo de absorção, pode sofrer discriminação entre as fontes de P, o que está de acordo com a observação de GUÉGUEN et al. (1968), ao admitirem que possa existir, após a passagem do fósforo para o sangue, discriminação metabólica do $\mathrm{P}$, em função de sua origem.

Com relação ao fato de a dieta controle apresentar maior conteúdo de retorno de $\mathrm{P}$ do osso para o trato digestivo $(\mathrm{P}<0,05)$, provavelmente, isto se deve à menor quantidade de $\mathrm{P}$ ingerida pelos animais desse tratamento. Nessa situação, de acordo com DeLUCA (1979), o organismo utiliza mecanismos, como reabsorção de $\mathrm{P}$ do osso, destinado a equilibrar o fósforo na corrente sangüínea.

Para o P reabsorvido dos demais tecidos que retorna para o trato gastrintestinal, o fosfato bicálcico foi semelhante ao Patos de Minas, monoamônio e supertriplo $(\mathrm{P}>0,05)$ e superior ao do Tapira, que, por sua vez, foi semelhante ao Patos de Minas, monoamônio e supertriplo. A dieta controle foi superior ao Tapira, Patos de Minas, monoamônio e supertriplo $(\mathrm{P}>0,05)$.

O P reabsorvido do osso e dos tecidos que retorna ao trato digestivo apresentou comportamento diferente para as fontes estudadas, o que pode ter ocorrido em função de o P em cada tecido desempenhar funções específicas nos processos metabólicos, exigindo maior ou menor participação do fósforo. À semelhança do que ocorreu para as quantidades de $\mathrm{P}$ ingerida, apesar de estatisticamente similares entre os tratamentos suplementados com as fontes de $\mathrm{P}$, os valores absolutos apresentaram variações, que podem ter contribuído para divergência na distribuição do $\mathrm{P}$ nos compartimentos estudados.

\section{Conclusões}

As hipóteses estabelecidas no modelo estão coerentes e os valores obtidos ajustam-se ao fluxo do fósforo no organismo de suínos em crescimento.

As variáveis $\mathrm{P}$ consumido, $\mathrm{P}$ de origem endógena que retorna ao trato gastrintestinal, $\mathrm{P}$ absorvido de origem dietética, retenção de $\mathrm{P}$, incorporação, reabsorção e balanço de $\mathrm{P}$ do osso e dos demais tecidos e $\mathrm{P}$ do total absorvido que retorna ao trato digestivo não foram influenciadas pelas fontes suplementares de fósforo.

O fósforo dos fosfatos avaliados apresenta alto valor biológico para suínos em crescimento, apresentando valores de 84,76; 73,03; 70,86; 85,63; e 75,83\%, respectivamente, para os fosfatos bicálcico, Tapira, Patos de Minas, monoamônio e supertriplo.

A origem do fósforo dietético pode interferir na distribuição do $\mathrm{P}$ nos tecidos, após o processo de absorção desse mineral.

OPdototal absorvidoque retorna ao tratogastrintestinal é proporcional à quantidade do $\mathrm{P}$ consumido.

\section{Referências Bibliográficas}

AMMERMAN, C.B., ARRIGTON, L.R., McCALL, J.T. et al. 1963. Inorganic phosphorus utilization by swine as measured by isotope technique. J. Anim. Sci., 22(4):890-893.

BARBOSA, H.P., MORES, N., FIALHO, E.T. et al. 1992. Efeitos de níveis de flúor provenientes dos fosfatos Tapira e mocálcico no desempenho e características do osso de suínos em crescimentos e terminação. R. Soc. Bras. Zootec., 21(5):838-846.

BELlAVER, C., GOMES, P.C., FIALHO, E.T. et al. 1984. Absorção e disponibilidade de fósforo de fosfatos naturais em rações para suínos. Pesq. Agropec. Bras., 19(12):1513-1515.

BASTIANELli, D., SAUVANT, D., RÉRAT, A. 1996. Mathematical modeling of digestion nad nutrient absorption in pigs. J. Anim. Sci., 74(10):1873-1887.

BROWN, D., ROTHERY, P. 1993. Models in biology: mathematics, statistics and computing. Chichester: John Wiley e Sons. 687p.

CARVALHO, F.F.R. Efeitos de níveis de fósforo sobre digestibilidade, metabolismo, perda endógena e cinética de fósforo em cabritos saanem. Jaboticabal, SP: UNESP 1998. 83 p. Tese (Doutorado) - Faculdade de Ciências Agrárias e Veterinárias, Universidade Estadual Paulista, 1998.

COMAR, C.L., MONROE, R.A., VISEK, W.J. et al. 1953. Comparison of two isotope methods for determination of endogenous fecal calcium. J. Nutr., 50:459-67.

DAVIDSOM, H.R. 1966. Pig meat: The carcass in relation to the live pig. In: DAVIDSOM, H.R. (Ed.) The production and marketing of pigs. London: Longmans. 3.ed. p.89-102.

DeLUCA, H.F. 1979. The vitamin D system in the regulation of calcium and phosphorus metabolism. Nutrition Review, 37(6):161-193.

ENGSTRON, G.W., HORST, R.L., REINHART, T.A. et al. 1985. Effect of dietary phosphorus levels on porcine renal 25-hydroxyvitamin D-1a and 24R-hidroxilase activities and plasma 1,25-dihydroxyvitamin $\mathrm{D}_{3}$ concentratition. J. Anim. Sci., 60(4):1005-1011.

FERNANDEZ, J.A. 1995. Calcium and phosphorus metabolism in growing pigs. III. A model resolution. Livest. Prod. Sci., 41(1):255-2261.

FIGUEIRÊDO, A.V. Disponibilidade biológica do fósforo de cincofosfatos, determinada em suínos em crescimento, através da técnica da diluição isotópica. Piracicaba, SP: USP. 
1998. 103p. Tese (Doutorado) - Centro de Energia Nuclear na Agricultura, Universidade de São Paulo, 1998.

FISHER L.J.A. 1978. Comparison of supplemental forms of phosphorus. Can. J. Anim. Sci., 58:313-317.

FURTADO, M.A.O. Determinação da biodisponibilidade de fósforo em suplementos de fósforo para aves e suínos. Belo Horizonte, MG: UFMG, 1991. 60p. Dissertação (Mestrado) - Universidade Federal de Minas Gerais. 1991.

GARCIA, C. 1997. Modelagem e simulação de processos industriais e de sistemas eletromecânicos. São Paulo: Editora da Universidade de São Paulo. 458p.

GOMES, P.C., BELLAVER, C., FIALHO, E.T. et al. 1985. Fontes alternativas de fósforo na alimentação de suínos em crescimento e terminação. R. Soc. Bras. Zootec., 14(2):241-246.

GOMES, P.C. Exigência nutricional de fósforo e sua disponibilidade em alguns alimentos para suínos de diferentes idades. Viçosa, MG: UFV, 1988. 163p. Tese (Doutorado) - Universidade Federal de Viçosa. 1988.

GOMES, P.C., LIMA, G.J.M.M., BARBOSA, H.P. et al. 1992. Disponibilidade de fósforo de Tapira e fosforindus e na farinha de ossso para suínos. R. Soc. Bras. Zootec., 21(1):83-89.

GRACE, N.D. 1981. Phosphorus kinetics in the sheeps. Brit. J. Nutr., 45:367-374.

GUÉGUEN, L., BESANÇON, P., RERAT, A. 1968. Utilization digestive, cinétique de lábsorption et efficacité de la rétention du phosphoro phytique chez le porc. Ann. Biol. Anim. Bioch. Biophys., 8(2):273-280.

INTERNATIONAL ATOMIC ENERGY AGENCY - IAEA. 1979. Laboratory training manual on the use of nuclear techniques in animal research. Vienna: IAEA. 299p. (Technical Report Series, 193).

KEBREAB, E., FRANCE, J. 1999. Biomathematical modelling guidelines for natural resources projects. Reading: University of Reading. 9p.

KeTAREN, P.P., BATTERHAM, E.S., White, E. 1993. Phosphorus studies in pig. 1. Available phosphorus requirements of grower/finisher pigs. Brit. J. Nutr., 70:249-268.

KLEIBER, M., SMITH, A.H., RALSTON, N.P. et al. 1951. Radiophosphorus $\left({ }^{32} \mathrm{P}\right)$ as tracer for measuring endogenous phosphorus in cow's feces. J. Nutr., 45(2):253-263.

LOPES, J.B. Avaliação da absorção real e da perdas endógenas de fósforo para suínos pela técnica de diluição isotópica. Piracicaba, SP: USP. 1998. 87p. Tese (Doutorado) - Centro de Energia Nuclear na Agricultura/Universidade de São Paulo, 1998.

LOPES, J.B. Efeitos dos níveis de proteína e energia sobre característica da carcaça de suínos em crescimento e terminação. In: SEMINÁRIO DE PESQUISA AGROPECUÁRIA DO PIAUÍ, 5, 1988, Teresina. Anais... Teresina: EMBRAPA, 1988. p.209-216.
LOPES, J.B., VITTI, D.M.S.S., ABDALLA, A.L. et al. Fluxo biológico do fósforo em suínos: uma simulação. (Compact disc). In: REUNIÃO ANUAL DA SOCIEDADE BRASILEIRA DE ZOOTECNIA, 36, 1999, Porto Alegre. Anais... Porto Alegre: SBZ, 1999 (NUN-091).

NATIONAL RESEARCH COUNCIL -NRC. 1998. Nutrient requirements of swine. 10.ed. Washington: National Academy of Science. 189p.

PEELER, H. T. 1972. Biological availability of nutrients in feeds: availability of major mineral ions. J. Anim. Sci., 35(3):695712.

PROCHÁZKA, Z., CUPÁK, M., JAMBOR, V. 1972. The utilization and distribution of phosphorus after peroral administration of ${ }^{32} \mathrm{P}$ labelled phosphorus compounds in pigs. Acta Vet. Brno, 41:263-270.

ROSTAGNO, H.S., SILVA, D.J., COSTA, P.M.A. et al. 1987. Composição de alimentos e exigências nutricionais de aves e suinos: tabelas brasileiras. Viçosa: UFV. 59p.

SAUVANT, D., BAUMONT, R., FAVERDIN, P. 1996. Development of a mechanistic model of intaake and chewing activities of sheep. J. Anim. Sci., 74(12):1785-2802.

SCHNEIDER, K.N., TERNOUTH, J.H., SEVILLA, C.C. et al. 1985. A shor-term study of calcium and phosphorus absorption in sheep fed on diets high and low incalcium and phosphorus. Aust. J. Agric. Res., 36:91-105.

SMITH, A.H., KLEIBER, M., BLACK, A.L. et al. 1951. Distribuition of intravenously injected radioative phosphorus $\left.{ }^{32} \mathrm{P}\right)$ among swine tissue. J. Anim. Sci., 10(4):893-901.

STATISTICAL ANALYSIS SISTEM. SAS. 1986. System for linear models. Cary: SAS Insitutte. 211p.

VITTI, D.M.S.S. Avaliação da disponibilidade biológica do fósforo dos fosfatos bicálcico, Patos de Minas, Tapira e finos de Tapira para ovinos pela técnica da diluição isotópica. São Paulo, SP: IPEN, 1989. 87p. Tese (Doutorado). Instituto de Pesquisas Energéticas Nucleares, 1989.

VITTI, D.M.S.S., ABDALLA, A.L., MEIRELLES, C.F. 1992. Cinética do fósforo em ovinos suplementados com diferentes fontes fosfatadas através da técnica de diluição isotópica. Arq. Bras. Med. Vet. Zootec., 44(3):227-233.

Recebido em: 19/04/00

Aceito em: 29/07/00 